\title{
On the modal logic of the iterated Cantor derivative and finitary operations on topological spaces
}

\author{
D. I. Saveliev* \\ Lomonosov Moscow State Univerity
}

We consider propositional language endowed with three families of modal operators:

$$
\{\langle\alpha\rangle: \alpha<\Omega\}, \quad\{\langle F\rangle: F \in \mathcal{F}\}, \quad\left\{\left(F^{-1}\right): F \in \mathcal{F}\right\},
$$

where $\Omega$ is an ordinal (or the class of ordinals) and $\mathcal{F}$ is a family of (unary or finitary) operations on a topological space. The natural semantics is as follows: the modality $\langle\alpha\rangle$ is interpreted by the $\alpha$ th iteration of the Cantor derivative, while the modalities $\langle F\rangle$ and $\left(F^{-1}\right)$ are interpreted by the image and the preimage of the operation $F$. We describe logics in this language that are sound w.r.t. their natural semantics. In particular, they express certain topological properties of operations, such as injectivity, continuity, opennes, closedness, continuity and discreteness, closedness and finite rank, separate continuity and separate discreteness (this is partially based on our recent work [1]). All these logics consist of Sahlqvist formulas, hence are complete, although not always w.r.t. their natural semantics.

We start with modalities $\langle\alpha\rangle$ for derivative operators. Recall that, for $X$ a topological space, $x \in X$ is a limit point of $A \subseteq X$ iff any neighborhood of $x$ contains a point $y \in A \backslash\{x\}$. The Cantor derivative operator takes each $A \subseteq X$ to its derived set $A^{\prime}=\{x \in X: x$ is a limit point of $A\}$. The closure $\operatorname{cl}(A)$ of a set $A$ is $A \cup A^{\prime}$. Iterations of the derivative are defined by $A^{(0)}=\operatorname{cl}(A), A^{(\alpha+1)}=\left(A^{(\alpha)}\right)^{\prime}$, and $A^{(\alpha)}=\bigcap_{\beta<\alpha} A^{(\beta)}$ if $\alpha$ is a limit ordinal. Thus the natural semantics of the modal operator $\langle\alpha\rangle$ is

$$
\|\langle\alpha\rangle \varphi\|=\|\varphi\|^{(\alpha)} .
$$

Recall that a space $X$ satisfies the $T_{d^{-}}$separation axiom iff any point of $X$ is the intersection of an open and a closed set. These spaces lie strictly between $T_{0^{-}}$and $T_{1}$-spaces and can be characterized e.g. as the spaces in which the derived set of every set is closed. Scattered spaces are always $T_{d}$. In the sequel, we shall assume that all our spaces are $T_{d}$ (although a part of the theory holds for $T_{0}$ as well).

Theorem 1. The logic consisting of the schemas

$$
\begin{aligned}
& \langle 0\rangle \varphi \leftrightarrow \varphi \vee\langle 1\rangle \varphi, \\
& \langle\alpha\rangle(\varphi \vee \psi) \leftrightarrow\langle\alpha\rangle \varphi \vee\langle\alpha\rangle \psi \text { for all } \alpha, \\
& \langle\beta\rangle\langle\alpha\rangle \varphi \leftrightarrow\langle\alpha+\beta\rangle \varphi \text { for all } \alpha, \beta,
\end{aligned}
$$

is sound w.r.t. all $T_{d}$-spaces.

This logic is complete.

Let us comment on this. (1.1) shows that $\langle 0\rangle$ can be defined via $\langle 1\rangle$. (1.3) states that the additive semigroup of ordinals acts by the operators $\langle\alpha\rangle$. It clearly follows that $\langle\alpha\rangle$ for all $\alpha<\omega$ can be defined via $\langle 1\rangle$. Moreover, only $\langle\alpha\rangle$ with additively indecomposable ordinals $\alpha$ cannot be

*Partly supported by RFBR grants 11-01-00958, 11-01-93107, and NSh grant 5593-2012-1. 
expressed via other $\langle\beta\rangle$ since these ordinals are generators of the additive semigroup of ordinals. Recall that an ordinal $\alpha$ is additively indecomposable iff $\beta+\gamma<\alpha$ for all $\beta, \gamma<\alpha$, which also holds iff $\beta+\alpha=\alpha$ for all $\beta<\alpha$, and iff $\alpha=0$ or $\alpha=\omega^{\xi}$ for some $\xi$. $\langle 0\rangle$ expresses the usual properties of closure: $\varphi \rightarrow\langle 0\rangle \varphi$ follows from (1.1), while $\langle 0\rangle \varphi \leftrightarrow\langle 0\rangle\langle 0\rangle \varphi$ is an instance of (1.3). If $\alpha>0$, then $\langle\alpha\rangle$ has the usual properties of derivative in $T_{d}$-spaces: (1.3) gives $\langle\beta\rangle \varphi \rightarrow\langle\alpha\rangle \varphi$ for all $\alpha \leq \beta$, and so implies $\langle\alpha\rangle\langle\alpha\rangle \varphi \rightarrow\langle\alpha\rangle \varphi$.

Note that all the axioms above are Sahlqvist, hence the completeness of the logic follows by the Sahlqvist theorem. However, the semantics of $\langle\alpha\rangle$ with $\alpha \geq \omega$ does not need to be as intended (e.g. $\langle\omega\rangle$ can be interpreted rather by the $(\omega+1)$ st than the $\omega$ th derivative). The natural interrelations between $\langle\alpha\rangle$ and $\langle\beta\rangle$ 's with $\beta<\alpha$ could be expressed by using infinitary connectives, but we did not consider here this possibility.

Let us now consider the modalities $\langle F\rangle$ and $\left(F^{-1}\right)$ intended to express the image and preimage. Thus their natural semantics is:

$$
\|\langle F\rangle(\varphi)\|=F(\|\varphi\|), \quad\left\|\left(F^{-1}\right)(\varphi)\right\|=F^{-1}(\|\varphi\|),
$$

where $F(A)$ and $F^{-1}(A)$ denote the image and the preimage of the set $A$ by the (unary) map $F$, and the axioms listed below are just as expected.

Theorem 2. The logic consisting of the schemas

$$
\begin{aligned}
& \langle F\rangle(\varphi \vee \psi) \leftrightarrow\langle F\rangle \varphi \vee\langle F\rangle \psi, \\
& \langle F\rangle(\varphi \wedge \psi) \rightarrow\langle F\rangle \varphi \wedge\langle F\rangle \psi, \\
& \left(F^{-1}\right)(\varphi \vee \psi) \leftrightarrow\left(F^{-1}\right) \varphi \vee\left(F^{-1}\right) \psi, \\
& \left(F^{-1}\right) \neg \varphi \leftrightarrow \neg\left(F^{-1}\right) \varphi, \\
& \langle F\rangle\left(F^{-1}\right) \varphi \leftrightarrow \varphi, \\
& \varphi \rightarrow\left(F^{-1}\right)\langle F\rangle \varphi, \\
& \langle G\rangle\langle F\rangle \varphi \leftrightarrow\langle G \circ F\rangle \varphi,
\end{aligned}
$$

is sound w.r.t. arbitrary unary operations.

This logic together with any of the schemas

(2.8) $\varphi \leftrightarrow\left(F^{-1}\right)\langle F\rangle \varphi$,

$$
\langle F\rangle(\varphi \wedge \psi) \leftrightarrow\langle F\rangle \varphi \wedge\langle F\rangle \psi
$$

is sound w.r.t. injective unary operations.

These logics are complete.

Of course, it follows from (2.3), (2.4) that the preimage modalities commute with every connectives and are self-dual (hence the notation using the round parentheses).

Again, the completeness here follows by the Sahlqvist theorem, and generally, this is not the completeness w.r.t. the natural semantics, although for the preimage modalities, it is.

Passing to finitary modal operators, we note that the preimages of finitary maps are essentially the preimages of corresponding unary maps, hence we shall interest only in the image operators $\langle F\rangle$. Their natural semantics is:

$$
\left\|\langle F\rangle\left(\varphi_{1}, \ldots, \varphi_{n}\right)\right\|=F\left(\left\|\varphi_{1}\right\|, \ldots,\left\|\varphi_{n}\right\|\right) .
$$


Given a property of unary maps (e.g. injectivity or continuity), let us say that a finitary map has this property separately iff each unary map obtained from it by fixing all but one argument has the property (e.g. in a group the multiplication is separately injective, and in a topological group it is separately continuous).

Theorem 3. The logic consisting of the schemas

$$
\begin{aligned}
&\langle F\rangle\left(\varphi_{1} \vee \psi_{1}, \ldots, \varphi_{n} \vee \psi_{n}\right) \leftrightarrow\langle F\rangle\left(\varphi_{1}, \ldots, \varphi_{n}\right) \vee\langle F\rangle\left(\psi_{1}, \ldots, \psi_{n}\right), \\
&\langle F\rangle\left(\varphi_{1} \wedge \psi_{1}, \ldots, \varphi_{n} \wedge \psi_{n}\right) \rightarrow\langle F\rangle\left(\varphi_{1}, \ldots, \varphi_{n}\right) \wedge\langle F\rangle\left(\psi_{1}, \ldots, \psi_{n}\right),
\end{aligned}
$$

is sound w.r.t. arbitrary n-ary operations.

This logic together with the schema

$$
\langle F\rangle\left(\varphi_{1} \wedge \psi_{1}, \ldots, \varphi_{n} \wedge \psi_{n}\right) \leftrightarrow\langle F\rangle\left(\varphi_{1}, \ldots, \varphi_{n}\right) \wedge\langle F\rangle\left(\psi_{1}, \ldots, \psi_{n}\right)
$$

is sound w.r.t. separately injective $n$-ary operations.

These logics are complete.

Concerning the completeness, recall that the Sahlqvist theorem holds for finitary modal operators as well, see [3].

Now we are going to produce logics combining topological modalities with modalities for operations. These logics extend basic logics $\mathcal{L}$ and $\mathcal{L}_{n}$, where $\mathcal{L}$ consists of schemas (1.1)-(1.3) and (2.1)-(2.7), while $\mathcal{L}_{n}$ consists of schemas (1.1)-(1.3), (3.1), and (3.2).

As usually, $F$ is continuous iff the preimages of open sets are open, open iff the images of open sets are open, closed iff the images of closed sets are closed, and discrete iff the preimages of points are (relatively) discrete. Let us also say that $F$ is of finite rank on all sets iff the preimages of points, intersected with any set, have a finite Cantor-Bendixson rank (for scattered spaces it is the same that a finite rank of all the preimages).

Theorem 4. The logic $\mathcal{L}$ together with any of the schemas

$$
\begin{aligned}
& \langle F\rangle\langle 0\rangle \varphi \rightarrow\langle 0\rangle\langle F\rangle \varphi \\
& \langle 0\rangle\left(F^{-1}\right) \varphi \rightarrow\left(F^{-1}\right)\langle 0\rangle \varphi
\end{aligned}
$$

is sound w.r.t. continuous unary operations.

The logic $\mathcal{L}$ together with the schema

$$
\langle\alpha\rangle\langle F\rangle \varphi \rightarrow\langle F\rangle\langle\alpha\rangle \varphi \text { for all } \alpha<\omega
$$

is sound w.r.t. closed unary operations.

The logic $\mathcal{L}$ together with the schema

$$
\left(F^{-1}\right)\langle\alpha\rangle \varphi \rightarrow\langle\alpha\rangle\left(F^{-1}\right) \varphi \text { for all } \alpha
$$

is sound w.r.t. open unary operations.

The logic $\mathcal{L}$ together with any of the schemas

$$
\begin{aligned}
& \langle F\rangle\langle\alpha\rangle \varphi \rightarrow\langle\alpha\rangle\langle F\rangle \varphi \text { for all } \alpha, \\
& \langle\alpha\rangle\left(F^{-1}\right) \varphi \rightarrow\left(F^{-1}\right)\langle\alpha\rangle \varphi \text { for all } \alpha
\end{aligned}
$$

is sound w.r.t. continuous discrete unary operations.

The logic $\mathcal{L}$ together with the schema 


$$
\langle\alpha\rangle\langle F\rangle \varphi \rightarrow\langle F\rangle\langle\alpha\rangle \varphi \text { for all } \alpha
$$

is sound w.r.t. closed unary operations of finite rank.

These logics are complete.

For the finitary case, notice that the closedness of an $n$-ary map $F$ means that the image $F\left(A_{1}, \ldots, A_{n}\right)$ is closed whenever so are all the $A_{1}, \ldots, A_{n}$, which is a weaker condition than the closedness of the corresponding unary map (i.e. the same map considered as unary).

Given ordinals $\alpha, \beta$, we denote by $\alpha \oplus \beta$ their (Hessenberg) natural sum, i.e. the ordinal written as the formal sum of the Cantor normal forms of $\alpha$ and $\beta$. E.g. $\left(\omega^{\omega}+\omega^{4} 2+1\right) \oplus\left(\omega^{6}+\omega^{4}\right)=$ $\omega^{\omega}+\omega^{6}+\omega^{4} 3+1$. Clearly, the natural addition is commutative and associative and dominates the usual ordinal addition.

Theorem 5. The logic $\mathcal{L}_{n}$ together with the schema

$$
\langle\alpha\rangle\langle F\rangle\left(\varphi_{1}, \varphi_{2}, \ldots, \varphi_{n}\right) \rightarrow \bigvee_{\alpha_{1} \oplus \ldots \oplus \alpha_{n}=\alpha}\langle F\rangle\left(\left\langle\alpha_{1}\right\rangle \varphi_{1},\left\langle\alpha_{2}\right\rangle \varphi_{2}, \ldots,\left\langle\alpha_{n}\right\rangle \varphi_{n}\right)
$$

is sound w.r.t. closed n-ary operations of finite rank.

The logic $\mathcal{L}_{n}$ together with the schema

$$
\bigvee_{\alpha_{1} \oplus \ldots \oplus \alpha_{n}=\alpha}\langle F\rangle\left(\left\langle\alpha_{1}\right\rangle \varphi_{1},\left\langle\alpha_{2}\right\rangle \varphi_{2}, \ldots,\left\langle\alpha_{n}\right\rangle \varphi_{n}\right) \rightarrow\langle\alpha\rangle\langle F\rangle\left(\varphi_{1}, \varphi_{2}, \ldots, \varphi_{n}\right)
$$

is sound w.r.t. separately continuous separately discrete n-ary operations.

These logics are complete.

Note that the disjunctions in the formulas above are finite; there are only finitely many ways to decompose any given ordinal into a natural sum of $n$ summands.

\section{References}

[1] D. I. Saveliev, On interactions of the Cantor derivative and images of finitary maps between topological spaces, 2012, submitted to Topology and its Applications.

[2] D. I. Saveliev, On modal logic of topological dynamics: actions of arbitrary semigroups, in: Th. Bolander et al. (eds.), 9th Advances in Modal Logic, Short Presentations, 2012, pp. 68-75.

[3] P. Blackburn, M. de Rijke, Y. Venema, Modal Logic, Cambridge Univ. Press, 2002. 